\section{Einfluss verschiedener Trainingsformen auf die Zellalterung und die kar- diovaskuläre Fitness}

\section{Originalpublikation}

Werner CM, Hecksteden A, Morsch A et al. Differential effects of endurance, interval, and resistance training on telomerase activity and telomere length in a randomized, controlled study. Eur Heart ] 2019; 40: 3446. doi:10.1093/eurheartj/ehy585

\section{Theoretischer Hintergrund}

Körperliches Training besitzt einen multidirektionalen positiven Einfluss auf die menschliche Gesundheit. Aufgrund dieser Effekte auf relevante Risikofaktoren für HerzKreislauf-Erkrankungen werden Kraft- und Ausdauertraining empfohlen, um das kardiovaskuläre Krankheitsrisiko zu verringern $[1,2]$. Ein Mangel an körperlicher Aktivität gilt als zentraler Risikofaktor für einen vorzeitigen Tod sowie für die Entwicklung von Herz-Kreislauf- und Stoffwechselerkrankungen. Regelmäßige körperliche Aktivität gilt somit als einer der wichtigsten Schutzfaktoren für die Gesundheit [3].

Für die Erhaltung und Förderung der Gesundheit werden pro Woche mindestens 150 Minuten aerobe körperliche Aktivität mit moderater Intensität oder 75 Minuten aerobe körperliche Aktivität mit höherer Intensität empfohlen [4]. Mehr als die Hälfte der deutschen Bevölkerung folgt diesen Empfehlungen jedoch nicht [5]. Neben aeroben Trainingsformen gilt das hochintensive Intervalltraining als effektive und zeiteffiziente Trainingsmethode zur Verbesserung der kardiorespiratorischen Fitness, die auch für Personen mit lebensstilbedingten Herz-Kreislauf- und Stoffwechselerkrankungen geeignet ist [6].

Aufgrund der insuffizienten Anzahl an randomisierten kontrollierten Vergleichsstudien konnte bislang nicht ausreichend geklärt werden, inwiefern sich verschiedene Trainingsformen hinsichtlich zellulärer Alterungseffekte unterscheiden [7]. Diese
Frage besitzt im Kontext der kardiovaskulären Gesundheit allerdings eine hohe Relevanz, da Zellalterung mit Funktionsstörungen des Endothels und mit Atherogenese assoziiert wird [8].

Die Verkürzung der Telomere wird als Schlüsselprozess der zellulären Alterung angesehen. Sie steht in engem Zusammenhang mit der verringerten Aktivität der Telomerase, die das Fortschreiten der Telomerlängenreduktion reguliert. Wird eine kritische Telomerlänge unterschritten, verliert die Zelle ihre Fähigkeit zur Teilung, wodurch ihre Regeneration zum Erliegen kommt. Dieser zelluläre Alterungsprozess wird als replikative Seneszenz bezeichnet und führt bei fortschreitender Telomerlängenreduktion zur Apoptose [9]. Auf Basis der beschriebenen Prozesse wird die Verringerung der Telomerlänge sowie der Telomeraseaktivität in mononukleären Blutzellen, wie beispielsweise Leukozyten, mit erhöhter kardiovaskulärer Mortalität in Verbindung gebracht $[10,11]$.

Es konnte bereits beobachtet werden, dass körperliche Aktivität einen positiven Einfluss auf die Telomeraseaktivität und die Telomerlängenreduktion haben kann. Allerdings war bisher keine verlässliche Aussage darüber möglich, inwiefern sich verschiedene Trainingsmodalitäten hinsichtlich ihrer Effekte auf zelluläre Alterungsprozesse voneinander unterscheiden [7].

\section{Untersuchungsmethodik}

Aufgrund des zuvor erläuterten Forschungsdefizits zielte die Studie von Werner, Hecksteden, Morsch et al. aus dem Jahr 2019 darauf ab, die Unterschiede von Trainingsformen hinsichtlich ihrer Langzeiteffekte auf Telomerlänge und Telomeraseaktivität von mononukleären Zellen bei gesunden Erwachsenen zu untersuchen [12].

Hierzu wurde eine prospektive, randomisierte Studie über 26 Wochen durchgeführt. Die Probandenstichprobe setzte sich dabei aus 266 gesunden, körperlich inaktiven erwachsenen Nichtraucher*innen im Alter zwischen 30-60 Jahren und mit einem BMI unter $30 \mathrm{~kg} / \mathrm{m} 2$ zusammen.
Unter Berücksichtigung des Geschlechts und der VO2max wurden die rekrutierten Personen randomisiert in vier Untersuchungsgruppen aufgeteilt, denen für die folgenden 26 Wochen jeweils eine Trainingsform zugeordnet wurde. Drei der vier Gruppen absolvierten innerhalb der Interventionsphase an drei Tagen pro Woche ein körperliches Training, wobei der Lebensstil und die Ernährung unverändert bleiben sollten. Dabei führte eine Gruppe (AET) ein 45-minütiges aerobes Ausdauertraining bei 60 Prozent der Herzfrequenzreserve aus. Die Proband* innen der zweiten Gruppe (IT) führten ein hochintensives Intervalltraining nach der $4 \times 4$-Methode durch. Die dritte Gruppe (RT) absolvierte ein gerätegestütztes Zirkeltraining zur Verbesserung der Kraftausdauer, das aus 8 Übungen für die großen Muskelgruppen des Körpers bestand. Die vierte Gruppe (control) diente als Kontrollgruppe und sollte ihr gewohntes Aktivitätsniveau während der Interventionsphase beibehalten. Im Untersuchungszeitraum schieden 119 Personen primär wegen mangelnder Einhaltung der Vorgaben aus der Studie aus. Weitere 23 Personen wurden aufgrund unvollständiger oder nicht plausibler Daten ausgeschlossen, wodurch insgesamt 124 Personen bei der Datenauswertung berücksichtigt werden konnten.

Zur Feststellung potenzieller Effekte auf die Telomerlänge und die Telomeraseaktivität wurden den Proband* innen vor Interventionsbeginn sowie zwischen 48 Stunden und 7 Tage nach der letzten Trainingseinheit Blutproben entnommen, aus denen mononukleäre Zellen (u. a. Leukozyten) zur weiteren molekularbiologischen Untersuchung isoliert wurden. Die anschließende inferenzstatistische Analyse zur Signifikanzprüfung der Ergebnisse erfolgte durch Berechnung von Varianzanalysen mit Messwiederholungen sowie t-Tests mittels SPSS.

\section{Ergebnisse}

Die 26-wöchige Trainingsintervention führte in allen 3 Trainingsgruppen zu einer signifikanten Verbesserung der körperlichen Fitness, gemessen anhand der $\triangle \mathrm{VO} 2 \mathrm{max}$ und der maximalen Laufgeschwindigkeit. 
Hinsichtlich der Telomerlängen von Lymphozyten und Granulozyten wurde nach 26 Wochen sowohl in der Kontrollgruppe (control) als auch in der Krafttrainingsgruppe (RT) eine leichte Reduktion beobachtet, während die Telomerlänge der Leukozyten keine Veränderung aufwies.

Im Gegensatz hierzu konnte in beiden Ausdauertrainingsgruppen (AET und IT) ein signifikanter Längenzuwachs bei den Telomeren von Lymphozyten, Granulozyten und Leukozyten ( $\mathbf{A} \mathbf{b b} \mathbf{b}$. 1a) beobachtet werden.

Im Hinblick auf die Telomeraseaktivität wurde nach 26 Wochen ebenfalls nur in den beiden Ausdauertrainingsgruppen (AET und IT) eine signifikante Verbesserung festgestellt, wohingegen sowohl in der Kontroll- (control) als auch in der Krafttrainingsgruppe (RT) keine signifikante Veränderung festgestellt werden konnte ( $>$ Abb. 1b). Die Veränderung der Telomeraseaktivität korrelierte in allen Gruppen mit der Veränderung der Telomerlänge der Lymphozyten.

Darüber hinaus konnte festgestellt werden, dass alle betrachteten Trainingsmodalitäten (AET, IT und RT) zu einer verstärkten Bildung von Schutzproteinen führten, die einem Telomerabbau vorbeugen.

Demzufolge besitzen sowohl Krafttraining als auch Ausdauertraining einen präventiven Effekt auf die Zellalterung, entweder durch die Bildung von Schutzproteinen oder durch die Erhöhung der Telomeraseaktivität.

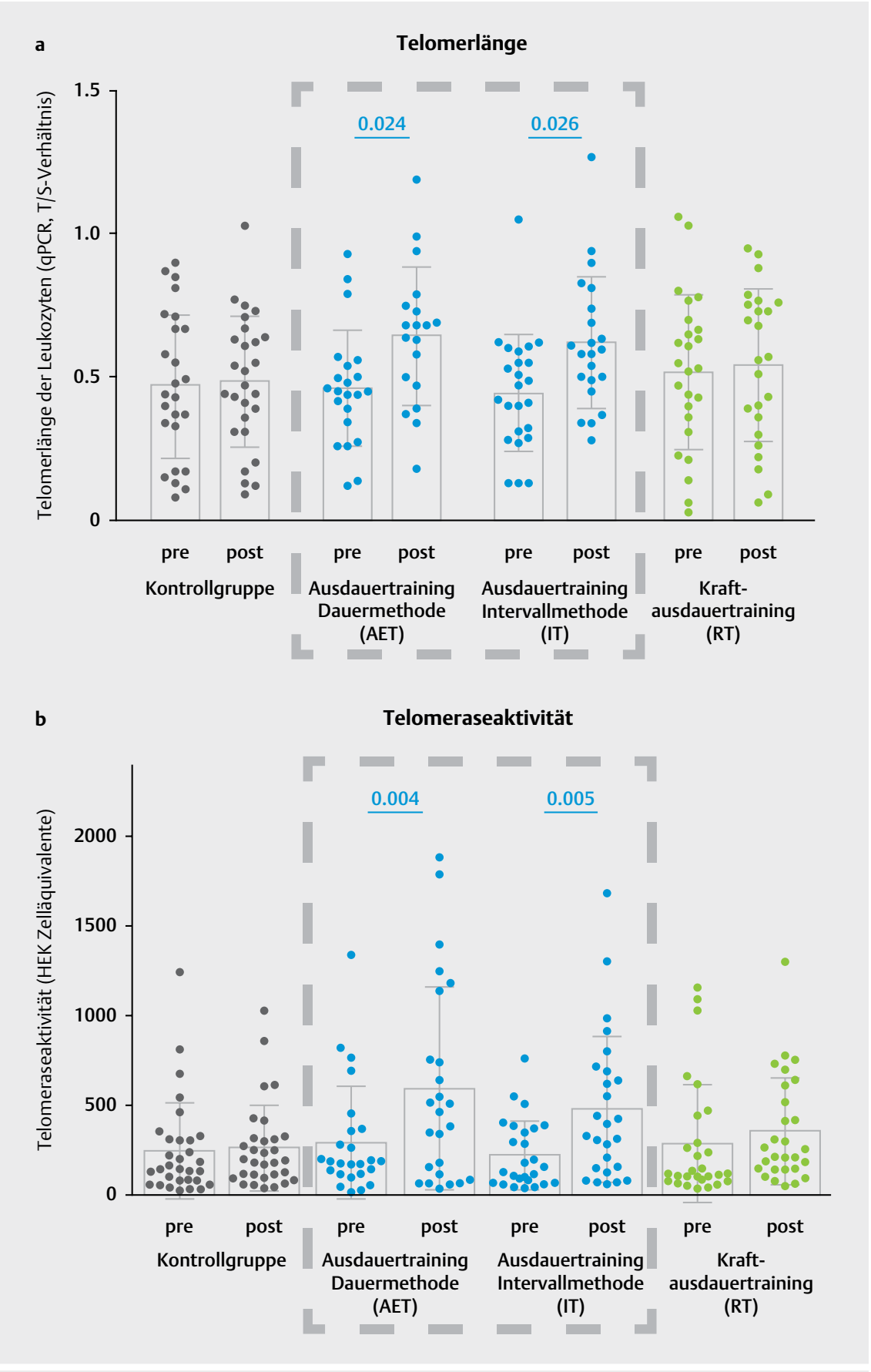

- Abb. 1 Differenzierte Effekte verschiedener Trainingsformen auf die Telomeraseaktivität und die Telomerlänge in zirkulierenden mononukleären Zellen. Quelle: Patrick Berndt mod. nach Werner, Hecksteden, Morsch et al. (2019) [12]. 
FAZIT

Entgegen der bisherigen Beobachtungen, dass körperliche Aktivität lediglich die Telomerlängenreduktion verlangsamt, konnte in der Studie von Werner, Hecksteden, Morsch et al. nachgewiesen werden, dass die Aufnahme und 26-wöchige Umsetzung eines Ausdauertrainings bei zuvor körperlich inaktiven Personen mittleren Alters zu einer Verlängerung der Telomere führt [12]. Dies kommt einer Umkehr des natürlichen Zellalterungsprozesses im Sinne eines Anti-Aging-Effekts gleich, wodurch die positive Wirkung des Ausdauertrainings auf die Herz-Kreislauf-Gesundheit untermauert wird.

Der zentrale Erkenntnisgewinn durch die vorgestellte Studie liegt in der Tatsache, dass Ausdauertraining sowohl die Telomeraseaktivität als auch die Telomerlänge von mononukleären Zellen positiv beeinflussen kann, unabhängig von der gewählten Ausdauertrainingsmethode. Diese Effekte konnten bei einem Kraftausdauertraining nicht beobachtet werden. Es kam jedoch zu einer gesteigerten Bildung von Schutzproteinen sowie zur Verbesserung der kardiorespiratorischen Fitness. Unter zusätzlicher Berücksichtigung der hier nicht erwähnten Effekte eines Krafttrainings für das Muskel-Skelett-System kann abschließend festgehalten werden, dass die größten gesundheitspositiven Effekte durch eine Kombination von Kraft- und Ausdauertraining zu erwarten sind.

\section{ZUSATZINFO}

\section{Funktion der Telomere}

Jede Zelle enthält einen Chromosomensatz, in dem ein Großteil der Erbinformation in Form von DNA gespeichert ist. Diese Information darf nicht beschädigt werden, damit die ordnungsgemäße Funktion der Zelle erhalten bleibt. Dabei schützen die Enden der Chromosomen, die als Telomere bezeichnet werden, die chromosomale DNA vor dem Abbau.

\section{Korrespondenzadresse}

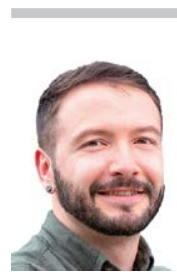

\section{Patrick Berndt}

Deutsche Hochschule für Prävention und Gesundheitsmanagement Hermann-NeubergerSportschule 3 66123

Saarbrücken

Deutschland info@dhfpg.de

\section{Literatur}

[1] Piepoli MF, Hoes AW, Agewall S et al. 2016 European Guidelines on cardiovascular disease prevention in clinical practice: The Sixth Joint Task Force of the European Society of Cardiology and Other Societies on Cardiovascular Disease Prevention in Clinical Practice (constituted by representatives of 10 societies and by invited experts). Developed with the special contribution of the European Association for Cardiovascular Prevention \& Rehabilitation (EACPR). Eur Heart J 2016; 37: 2315-2381. doi:10.1093/ eurheartj/ehw106

[2] Schuler G, Adams V, Goto Y. Role of exercise in the prevention of cardiovascular disease: results, mechanisms, and new perspectives. Eur Heart J 2013; 34: 1790-1799. doi:10.1093/eurheartj/eht111
[3] Kraus WE, Powell KE, Haskell WL et al. Physical Activity, All-Cause and Cardiovascular Mortality, and Cardiovascular Disease. Med Sci Sports Exerc 2019; 51: 1270-1281. doi:10.1249/MSS.0000000000001939

[4] Pfeifer K, Rütten A. Nationale Empfehlungen für Bewegung und Bewegungsförderung. Gesundheitswesen 2017; 79: S2-S3. doi:10.1055/s-0042-123346

[5] Finger JD, Mensink GBM, Lange C et al. Gesundheitsfördernde körperliche Aktivität in der Freizeit bei Erwachsenen in Deutschland. J Health Monit 2017; 2: 37-44. doi:10.17886/RKI-GBE-2017-027

[6] Weston KS, Wisløff U, Coombes JS. High-intensity interval training in patients with lifestyle-induced cardiometabolic disease: a systematic review and metaanalysis. Br J Sports Med 2014; 48: 1227-1234. doi:10.1136/ bjsports-2013-092576

[7] Mundstock E, Zatti H, Louzada FM et al. Effects of physical activity in telomere length: Systematic review and meta-analysis. Ageing Res Rev 2015; 22: 72-80. doi:10.1016/j.arr.2015.02.004

[8] López-Otín C, Blasco MA, Partridge L et al. The hallmarks of aging. Cell 2013; 153 : 1194-1217. doi:10.1016/j.cell.2013.05.039

[9] Xie Z, Jay KA, Smith DL et al. Early Telomerase Inactivation Accelerates Aging Independently of Telomere Length. Cell 2015; 160: 928-939. doi:10.1016/j. cell.2015.02.002

[10] Brouilette SW, Moore JS, McMahon AD et al. Telomere length, risk of coronary heart disease, and statin treatment in the West of Scotland Primary Prevention Study: a nested case-control study. Lancet 2007; 369: 107-114. doi:10.1016/S01406736(07)60071-3

[11] Wong LSM, Boer de RA, Samani NJ et al. Telomere biology in heart failure. Eur ] Heart Fail 2008; 10: 1049-1056. doi:10.1016/j.ejheart.2008.08.007

[12] Werner CM, Hecksteden A, Morsch A et al. Differential effects of endurance, interval, and resistance training on telomerase activity and telomere length in a randomized, controlled study. Eur Heart ] 2019; 40: 34-46. doi:10.1093/eurheartj/ ehy 585 